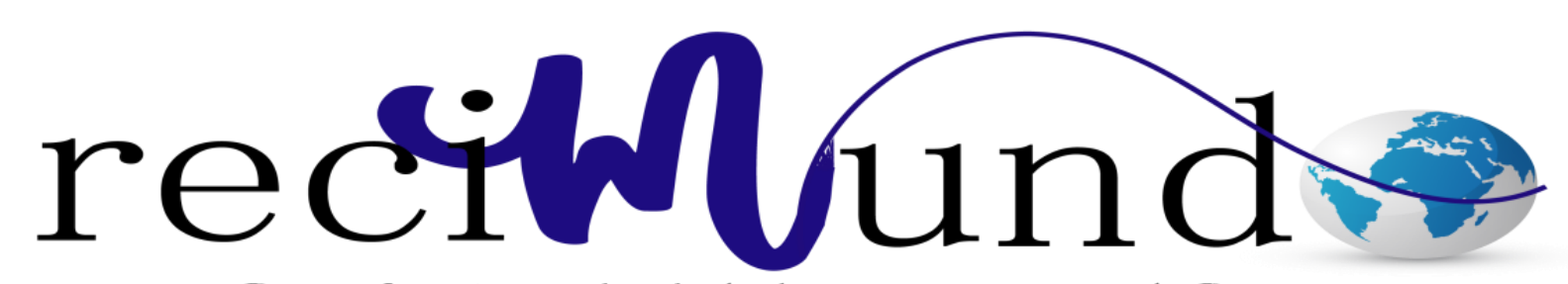

Revista Científica Mundo de la Investigación y el Conocimiento

Genesis Carolina Perez Cruz a ${ }^{\text {; }}$ Elizabeth Stefania Molina Vega ${ }^{\text {b }}$ Raquel Alexandra Colcha Gonzales ${ }^{c}$

Aplicación del test de Denver II en la evaluación del desarrollo infantil

Application of de Denver II test in the evaluation of child development

Revista Científica Mundo de la Investigación y el Conocimiento. Vol. 3 núm.3. Esp., noviembre, ISSN: 2588-073X, 2019, pp. 25-37

DOI: $10.26820 /$ recimundo/3.(3.Esp).noviembre.2019.25-37

URL: http://recimundo.com/index.php/es/article/view/600

Código UNESCO: 3205 Medicina Interna

Tipo de Investigación: Artículo de Revisión

(C) RECIMUNDO; Editorial Saberes del Conocimiento, 2019

Recibido: 15/09/2019

Aceptado: 23/10/2019

Publicado: 30/11/2019

Correspondencia: genesisperez0926@gmail.com

a. Médico; Investigadora Independiente; Guayaquil, Ecuador; genesisperez0926@gmail.com

b. Médico; Investigadora Independiente; Guayaquil, Ecuador.

c. Médico; Investigadora Independiente; Guayaquil, Ecuador. 


\section{Aplicación del test de Denver II en la evaluación del desarrollo infantil \\ Vol. 3, núm. 3 Esp., (2019) \\ Genesis Carolina Perez Cruz; Elizabeth Stefania Molina Vega; Raquel Alexandra Colcha Gonzales}

\section{RESUMEN}

Entre las pruebas de tamizaje más usadas a nivel mundial para examinar los progresos en el desarrollo de niños desde el momento de su nacimiento hasta los seis años de edad, se encuentra el Test de Denver II (DDST - II), el mismo evalúa cuatro áreas del desarrollo del niño: PersonalSocial, Motor Fino-Adaptativo, Lenguaje, Motor Grande. El objetivo fundamental de la presente investigación es plasmar lo relacionado con el Test de Denver II: generalidades, ventajas, materiales, recomendaciones y normativa para su aplicación. El diseño de investigación que se llevó a cabo fue de tipo documental o bibliográfico. El DDST-II fue creado con la finalidad de diagnosticar retrasos en el desarrollo, mediante la adaptabilidad del sistema nervioso central llamada plasticidad cerebral. "La fiabilidad del test es del 90\% y entre evaluadores del 80 - 95\% es de fácil aplicación y no es costoso, su sensibilidad es de 56 al $83 \%$ y una especificidad entre el 43 al 80\%". Se concluye que el abordaje temprano de la mayoría de los problemas de desarrollo infantil mejora significativamente su pronóstico, por tanto, la aplicación de este tipo de test como lo es el DDST II permiten que el niño que presente alguna patología del desarrollo logre ser tratado precozmente y aumente sus posibilidades de que tenga una mejor calidad de vida.

Palabras Claves: Test; Denver; Evaluación; Desarrollo; Infantil. 


\title{
Aplicación del test de Denver II en la evaluación del desarrollo infantil
}

Vol. 3, núm. 3 Esp., (2019)

Genesis Carolina Perez Cruz; Elizabeth Stefania Molina Vega; Raquel Alexandra Colcha

Gonzales

\begin{abstract}
Among the screening tests most used worldwide to examine the progress in the development of children from the moment of birth to six years of age, is the Denver II Test (DDST - II), it evaluates four areas of the child's development: Personal-Social, Fine-Adaptive Motor, Language, Large Motor. The fundamental objective of the present investigation is to capture what is related to the Denver II Test: generalities, advantages, materials, recommendations and regulations for its application. The research design that was carried out was documentary or bibliographic. DDST-II was created with the purpose of diagnosing developmental delays, through the adaptability of the central nervous system called cerebral plasticity. "The reliability of the test is $90 \%$ and between evaluators $80-95 \%$ is easy to apply and is not expensive, its sensitivity is 56 to $83 \%$ and a specificity between 43 to $80 \%$." It is concluded that the early approach to most of the problems of child development significantly improves their prognosis, therefore, the application of this type of test such as DDST II allows the child who presents some developmental pathology to be treated early and increase your chances of having a better quality of life.
\end{abstract}

Keywords: Test; Denver; Evaluation; Development; Child. 


\section{Aplicación del test de Denver II en la evaluación del desarrollo infantil \\ Vol. 3, núm. 3 Esp., (2019) \\ Genesis Carolina Perez Cruz; Elizabeth Stefania Molina Vega; Raquel Alexandra Colcha Gonzales}

\section{Introducción.}

El Desarrollo del niño, sobre todo durante sus primeros años de vida, es uno de los aspectos fundamentales a considerar en la evaluación médica, ya que es allí donde el crecimiento es mucho más rápido y es donde el sistema nervioso crece y se desarrolla como en ninguna otra etapa de la vida del ser humano.

Los niños tienen controles pediátricos, llamados control de niños sanos, con mucha frecuencia en los primeros años de su vida, motivado a los importantes y acelerados cambios que ocurren durante el desarrollo de su cuerpo en esta etapa. Estos controles incluyen un examen físico completo, donde el médico verifica el crecimiento y desarrollo del niño a los fines de encontrar o prevenir cualquier problema. (Enciclopedia Médica ADAM, 2019)

"El retraso global del desarrollo afecta 1-3\% de los niños, cerca del 1\% tiene un trastorno del espectro autista, 1-2\% una discapacidad de aprendizaje leve, 0,3-0,5\% una discapacidad de aprendizaje severa, 5-10\% discapacidad específica de aprendizaje en un solo dominio". Es importante considerar que los niños presentan diferentes ritmos de desarrollo, por lo tanto, resulta fundamental distinguir a aquellos que están dentro de un rango normal de aquellos que están siguiendo un patrón patológico. En la actualidad se ha evidenciado que la detección e intervención tempranas son un factor primordial para el alcance de mejores resultados de los niños que presentan alteraciones en su desarrollo. (Bellman, Byrne, \& Sege, 2015).

Son múltiples las pruebas de tamiz o cribado en todo el mundo, que pueden ser usadas con la finalidad de evaluar el crecimiento y desarrollo del niño desde su nacimiento. Dichas pruebas permiten emitir un diagnostico a niños sanos, con el objeto de distinguirlos de los que no 


\section{Aplicación del test de Denver II en la evaluación del desarrollo infantil}

Vol. 3, núm. 3 Esp., (2019)

Genesis Carolina Perez Cruz; Elizabeth Stefania Molina Vega; Raquel Alexandra Colcha Gonzales

lo están. El objetivo fundamental de este diagnóstico o diferenciación consiste en la detección precoz de enfermedades a los fines de mejorar su pronóstico, por ende, cualquier posibilidad de muerte prematura del infante, así como cualquier tipo de discapacidad que se encuentre asociada a la enfermedad detectada.

García Pérez \& Martínez Granero (2016) mencionan en su trabajo la recomendación de La Academia Americana Pediatría de que "en cada visita de control del niño sano detectar factores de riesgo. Si los hay, aplicar herramienta de cribado. Si no los hay, solo se aplican en las visitas de 9, 18 y 24-30 meses". Asimismo, los autores refieren que la recomendación en general se debe centrar en mantener la vigilancia en todas las revisionesque se le realicen al niño. (p. 89)

Para Rizzoli et al. (2015) "una prueba de tamiz busca identificar a los individuos presuntamente enfermos dentro de una población aparentemente sana y establecer el riesgo de retraso de estos". Estas pruebas deben ser fáciles y rápidas de aplicar, confiables, económicamente viables, además de estar validadas de acuerdo al estándar de oro que permita conocer su sensibilidad y especificidad, que debería superar al 70\%, todo ello a los fines de ser verdaderamente útiles en el desarrollo infantil temprano. Es importante destacar que en todo el mundo existen muchas y variadas pruebas de tamiz validadas. (p. 377)

La Comisión Nacional de Protección Social en Salud de México (2013) en su Manual para la Evaluación de Menores de Cinco Años con Riesgo de Retraso en el Desarrollo, menciona como ejemplos de tamizaje que han sido validadas en diversos países del mundo:

El Denver (DDST y DDST II) (Frankenburg, 1971; Glascoe, 1992) y Ages and Stages Questionnaries (A\&SQ) (Squires, 1997; Gollenberg, 2010). En Latinoamérica en el 


\section{Aplicación del test de Denver II en la evaluación del desarrollo infantil}

Vol. 3, núm. 3 Esp., (2019)

Genesis Carolina Perez Cruz; Elizabeth Stefania Molina Vega; Raquel Alexandra Colcha Gonzales

2009, en Chile se validó A\&SQ utilizando como estándar de referencia la escala de desarrollo Bayley Scale of Infant Development III (BSID) encontrando una correlación entre ambas pruebas de 0.52 , con una sensibilidad de A\&SQ de 58.8\% y una especificidad de 87.2\% (Schonhaut, 2009); y en Argentina la Prueba Nacional de Pesquisa (Pascucci, 2002). (p. 19)

Entre las pruebas de tamizaje más usadas a nivel mundial para examinar los progresos en el desarrollo de niños desde el momento de su nacimiento hasta los seis años de edad, se encuentra el Test de Denver (DDST - por sus siglas en inglés Denver Developmental Screening Test), nombrado así porque fue creado en el Centro Médico de la Universidad de Colorado en Denver en el año 1967 y del cual actualmente se usa su versión mejorada: el DDST-II. Esta prueba evalúa cuatro áreas del desarrollo del niño: Personal-Social, Motor Fino-Adaptativo, Lenguaje, Motor Grande.

El propósito fundamental de la presente investigación consiste en revisar y plasmar lo concerniente al DDST-II, sus generalidades, ventajas, materiales, recomendaciones y normativa para su aplicación.

\section{Materiales y Métodos.}

Se usaron variadas fuentes documentales para la localización de los documentos bibliográficos. Se realizó una búsqueda bibliográfica aleatoria y consecutiva, desde el año 2013 hasta el 2019, en las siguientes bases de datos: MedlinePlus, Biblioteca Virtual de la Salud (BVS), PubMed, SciELO, Dialnet, Asociación Española de Pediatría, entre otras. Se utilizaron los descriptores: “Test Denver", “Test Denver II", “Aplicación Test Denver II", “DDST-II 


\section{Aplicación del test de Denver II en la evaluación del desarrollo infantil}

Vol. 3, núm. 3 Esp., (2019)

Genesis Carolina Perez Cruz; Elizabeth Stefania Molina Vega; Raquel Alexandra Colcha Gonzales

abstract" y "Pruebas de Tamiz o Cribado para evaluar desarrollo psicomotor del niño". Los registros obtenidos oscilaron entre 100 y 12 registros tras la combinación de las diferentes palabras clave. Asimismo, se realizó una búsqueda en internet en el buscador "Google académico" con los mismos términos.

Fueron seleccionados aquellos documentos contentivos de información relacionada específicamente con la aplicación del Test de Denver II en la evaluación del desarrollo infantil, sus generalidades, materiales, cómo se administra la prueba, componentes y normas para su aplicación.

\section{Resultados.}

Test de Denver (DDST-II) - Generalidades

Rivera \& Salto (2017) refieren que el test de Denver fue desarrollado por William K. Frankenburg y J.B. Dobbs en 1967 y creado en el Centro Médico de la Universidad de Colorado en Denver. El mismo evalúa cuatro áreas del desarrollo de los niños: Personal-Social, Motor Fino-Adaptativo, Lenguaje y Motor Grueso. Se aplica a niños de cero hasta los seis años. Este test fue creado con la finalidad de diagnosticar retrasos en el desarrollo, mediante la adaptabilidad del sistema nervioso central llamada plasticidad cerebral. "La fiabilidad del test es del $90 \%$ y entre evaluadores del $80-95 \%$ es de fácil aplicación y no es costoso, su sensibilidad es de 56 al 83\% y una especificidad entre el 43 al 80\%”. El test de Denver II ha sido estudiado en una población estratégica con respecto a la cultura, etnia, lugar de residencia, educación materna, no requiere un nivel de lectura y se encuentra dirigido básicamente hacia los niños. (p. 30, 31) 


\section{Aplicación del test de Denver II en la evaluación del desarrollo infantil}

Vol. 3, núm. 3 Esp., (2019)

Genesis Carolina Perez Cruz; Elizabeth Stefania Molina Vega; Raquel Alexandra Colcha Gonzales

El tamiz Denver II no mide la inteligencia, si no la diferencia entre sujetos regulares y aquellos que presentan alguna dificultad en el desarrollo con el objeto de brindar una oportuna intervención y evitar, en la medida de lo posible, efectos secundarios asociados con alguna enfermedad. Es una prueba evolutiva, constante de 125 reactivos los cuales están formados en cuatro grupos: Personal-Social, Motor Fino-Adaptativo, Lenguaje y Motor Grande. El grupo de los reactivos Personal- Social, se refieren a la conducta del niño en relación con otras personas, la socialización con el medio que lo rodea y la relación con él mismo. El Motor Fino-Adaptativo, contiene la una evaluación ojo-mano, es decir, está relacionado con aquellas habilidades de coordinación, concentración y destrezas manuales del niño, tales como la pinza manual. El grupo de Lenguaje engloba las habilidades para escuchar y comunicarse a través del habla o bien mediante gestos. En el grupo de Motor Grande, se encuentran las habilidades, movimientos, coordinación y control de los segmentos del cuerpo tales como: piernas, brazos, tronco, entre otros. (Rivera \& Salto, 2017, p. 31, 32)

Para Vicente, Pérez, Gavín, \& Martín (2016) “establecer un diagnóstico de certeza de trastorno del desarrollo en los primeros años de la vida es a veces complicado debido a que los límites de la normalidad son imprecisos", esto motivado a que pueda existir una variante de la normalidad o situaciones transitorias y benignas, por lo tanto, resulta importante considerar y conocer el curso evolutivo del desarrollo del niño. (p. 18).

\section{Ventajas}

Para Barreto Solis (2017) la prueba tiene las siguientes ventajas: 


\section{Aplicación del test de Denver II en la evaluación del desarrollo infantil}

Vol. 3, núm. 3 Esp., (2019)

Genesis Carolina Perez Cruz; Elizabeth Stefania Molina Vega; Raquel Alexandra Colcha Gonzales

- Una buena confiabilidad en la aplicación y reaplicación (correlación superior a 0.90 cuando se aplica varias veces).

- Es una medida estandarizada que ha sido normada en una muestra diversa.

- Puede ser administrado rápidamente por profesionales y paraprofesionales entrenados.

- Puede ser una herramienta de tamizaje útil cuando se utiliza con criterio clínico.

- El manual de entrenamiento establece como debe conjugarse el juicio clínico con el conocimiento del sistema de salud local, antes de referir a un niño sospechoso. (p. 28)

\section{Recomendaciones}

Esta prueba se administra de forma individual y su tiempo de duración es de aproximadamente 20 minutos. Algunas recomendaciones para aplicar la prueba serían crear y mantener un ambiente agradable para el niño, donde se encuentre presente la familia para brindarle seguridad y tranquilidad, que son fundamentales al momento de evaluar. A pesar de que no es necesario realizar la prueba en un orden estricto, algunos autores sugieren comenzar por el área de socialización, lo importante es valorar cada situación particular. Asimismo, es importante considerar antes de la evaluación que el niño esté sano, no tenga ningún malestar, ni se encuentre hambriento o con sueño. 


\section{Aplicación del test de Denver II en la evaluación del desarrollo infantil}

Vol. 3, núm. 3 Esp., (2019)

Genesis Carolina Perez Cruz; Elizabeth Stefania Molina Vega; Raquel Alexandra Colcha Gonzales

\section{Material para realizar las pruebas}

Los principales materiales a ser usados durante el test son un sonajero de colores vistosos, algunos juguetes, muñecos, coche, figuras, también se usan cubos, pelota, paño de tela, juegos de encajar vaso. Igualmente se debe disponer de dibujos de objetos de uso común, papel, lápiz y bolígrafo.

Cómo se debe aplicar la prueba

Básicamente las normas indican cuando el 25\%, el 50\%, el 75\%, y el 90\% de niños pasan cada tarea. Esta evaluación del desarrollo del niño se fundamenta en el desempeño del niño y en reportes ofrecidos por sus padres relacionados con cuatro áreas de funcionamiento que comprenden: motor fino, motor grueso, personal social y habilidad de lenguaje.

(Prieto Muñóz, 2015) explica la forma de administrar la prueba de la siguiente manera:

1. El examinador traza una línea (línea de edad) sobre la hoja de la prueba que una la edad del niño en ambas escalas.

2. Colocar en la parte superior de la línea trazada el nombre del examinador con la fecha de la evaluación.

3. Debe evaluar todas aquellas tareas que sean atravesadas por la línea de edad o bien que estén ligeramente atrás de ésta si antes no han sido evaluadas.

4. Procede a evaluar las tareas seleccionadas, dependiendo del resultado coloca cualquiera de las siguientes claves sobre la marca del 50\% de la población de referencia: 


\section{Aplicación del test de Denver II en la evaluación del desarrollo infantil}

Vol. 3, núm. 3 Esp., (2019)

Genesis Carolina Perez Cruz; Elizabeth Stefania Molina Vega; Raquel Alexandra Colcha Gonzales

a. P (pasó) si el niño realiza la tarea.

b. F (falló) si el niño no realiza una tarea que la hace el $90 \%$ de la población de referencia.

c. NO (nueva oportunidad) si el niño no realiza la prueba, pero aún tiene tiempo para desarrollarla (la línea de edad queda por detrás del 90\% de la población de referencia).

d. R (rehusó), el niño por alguna situación no colaboró para la evaluación, automáticamente se convierta en una NO porque hay que evaluar en la siguiente visita. (p. 20, 21).

Para Alcántara Rodríguez (2017) "una prueba se considera como ANORMAL cuando hay uno o más sectores con dos o más fallos y DUDOSA cuando hay dos o más sectores con un solo fallo". Es fundamental destacar que aquellos niños que no realizan o cumplen una tarea y su edad se encuentra entre el $75^{\circ}$ y $90^{\circ}$ percentil, se consideran en riesgo y es urgente que se implementen para estas intervenciones con actividades que favorezcan su desarrollo y que estén acordes a su edad.

\section{Conclusiones.}

La evaluación del desarrollo del niño desde etapas muy temprana y de la incorporación a cada etapa de pautas madurativas en concordancia con su edad resultan factores imprescindibles en la práctica del pediatra, por cuanto es por medio de esta valoración que se podrá detectar cualquier alteración que de alguna manera influya negativamente en el desempeño futuro del niño. 


\section{Aplicación del test de Denver II en la evaluación del desarrollo infantil}

Vol. 3, núm. 3 Esp., (2019)

Genesis Carolina Perez Cruz; Elizabeth Stefania Molina Vega; Raquel Alexandra Colcha Gonzales

Es la atención primaria la encargada de prestar especial atención a las inquietudes, planteamientos y preocupaciones de los padres, asimismo de llevar una historia clínica completa del niño y realizar el examen físico exhaustivos y necesarios para favorecer una atención especializada temprana en caso de cualquier sospecha de alteración de su desarrollo.

El Test de Denver II persigue determinar las capacidades de los niños en base a la edad en la que se encuentren, y esto a su vez, es una herramienta importante en el diagnóstico temprano de deficiencias en el niño evaluado. Por último, el abordaje temprano de la mayoría de los problemas de desarrollo infantil mejora significativamente su pronóstico, por tanto, la aplicación de este tipo de test como lo es el DDST II permiten que el niño que presente alguna patología del desarrollo logre ser tratado precozmente y aumente sus posibilidades de que tenga una mejor calidad de vida.

\section{Bibliografía.}

Alcántara Rodríguez, F. D. (2017). Retraso psicomotriz de los niños que acuden al Centro Infantil Municipal $N^{\circ} 2$ del Mercado Centro Comercial Loja y su relación con los factores de riesgo. UNIVERSIDAD NACIONAL DE LOJA, FACULTAD DE LA SALUD HUMANA. LOJA: UNIVERSIDAD NACIONAL DE LOJA.

Barreto Solis, E. L. (2017). Funcionamiento familiar y desarrollo psicomotriz en niños del centro infantil San Alfonso Riobamba, periodo diciembre 2016- mayo 2017. FACULTAD DE CIENCIAS DE LA SALUD, CARRERA DE PSICOLOGÍA CLÍNICA. Riobamba: Universidad Nacional de Chimborazo.

Bellman, M., Byrne, O., \& Sege, R. (25 de Septiembre de 2015). Evaluación del desarrollo en niños. Obtenido de IntraMed: https://www.intramed.net/contenidover.asp?contenidoid=79042

Comisión Nacional de Protección Social en Salud de México. (2013). Manual para la evaluación de menores de cinco años. Unidad de Investigación en Neurodesarrollo (UIN) del Hospital Infantil de, Secretaría de Salud. México D.F.: Primera Edición. 


\section{Aplicación del test de Denver II en la evaluación del desarrollo infantil}

Vol. 3, núm. 3 Esp., (2019)

Genesis Carolina Perez Cruz; Elizabeth Stefania Molina Vega; Raquel Alexandra Colcha

Gonzales

Enciclopedia Médica ADAM. (02 de Octubre de 2019). Control del niño sano. Obtenido de Medline Plus: https://medlineplus.gov/spanish/ency/article/001928.htm

García Pérez, M. A., \& Martínez Granero, M. A. (2016). Desarrollo psicomotor y signos de alarma. Asociación Española de Pediatría, Hospital Universitario Fundación Alcorcón Neuropediatría. Madrid: Lúa Ediciones.

Prieto Muñóz, K. P. (2015). Detección de alteraciones del neurodesarrollo mediante exámen clínico de Amiel-Tison y aplicación del test de Denver en niños de 24 meses en Machala. UNIVERSIDAD NACIONAL DE LOJA, ÁREA DE LA SALUD HUMANA. Loja: Universidad Nacional de Loja.

Rivera, M., \& Salto, D. (2017). Screening del desarrollo psicomotor en niños y niñas que asisten a los centros infantiles del buen vivir Patamarca II Y Hermann Gmeiner en el periodo del año 2017. Tesis de Grado, Facultad de Ciencias Médicas, Escuela de Tecnología Médica, Ecuador.

Rizzoli, A., Ismael Delgado-Ginebraa, L. A.-O.-H.-P., Villasís, M., Reyes, H., O'Shea, G., Aceves, D. C., . . . Muñóz , O. (2015). Impacto de un modelo de capacitación de la prueba Evaluación del Desarrollo Infantil en la atención primaria. Boletín Médico del Hospital Infantil de México, 72(3), 376-384.

Vicente, M., Pérez, A., Gavín, P., \& Martín, J. (2016). Programa de actividades de detección precoz de problemas de salud entre los 0 y 14 años: Efectividad, seguridad y evaluación económica de las diferentes alternativas existentes en la detección precoz de las alteraciones del desarrollo psicomotor. Ministerio de Sanidad, Servicios Sociales e Igualdad de España, Informes de Evaluación de Tecnologías Sanitarias: IACS. Madrid: Ministerio de Sanidad España e Instituto Aragonés de Ciencias de la Salud.

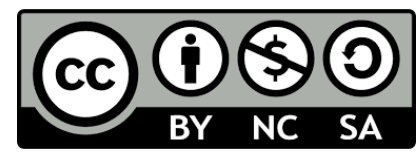

RECONOCIMIENTO-NOCOMERCIAL-COMPARTIRIGUAL

CC BY-NC-SA

ESTA LICENCIA PERMITE A OTROS ENTREMEZCLAR, AJUSTAR Y CONSTRUIR A PARTIR DE SU OBRA CON FINES NO COMERCIALES, SIEMPRE Y CUANDO LE RECONOZCAN LA AUTORÍA Y SUS NUEVAS CREACIONES ESTÉN BAJO UNA LICENCIA CON LOS MISMOS TÉRMINOS. 